\title{
ON THE IDEAL STRUCTURE OF $C(X)$
}

\author{
BY \\ WILLIAM E. DIETRICH, JR.(1)
}

\begin{abstract}
The ideal structure of $C(X)$, the algebra of continuous functions from a completely regular Hausdorff space $X$ to the scalars is analyzed by examining for fixed $A \subset \beta X$ (the Stone-Cech compactification of $X$ ) the structure of the quotient $I^{A} / F^{A}$, where $I^{A}\left[F^{A}\right]$ is the ideal of maps $f \in C(X)$ for which

$$
A \subset \mathrm{cl}_{\beta X} Z(f) \quad\left[A \subset \operatorname{int}_{\beta X} \mathrm{cl}_{\beta X} Z(f)\right] .
$$

Unless it vanishes, $I^{A} / F^{A}$ has no minimal or maximal ideals, and its Krull dimension is infinite. If $J$ is an ideal of $C(X)$ strictly between $F^{A}$ and $I^{A}$, there are ideals $\underline{J}$ and $\bar{J}$ of $C(X)$ for which $F^{A} \subset \underline{J} \subset J \subset \bar{J} \subset I^{A}$ with all inclusions proper. For $K \subset C(X)$, let $Z(K)=\cap\left\{\mathrm{cl}_{\beta X} Z(f): f \in K\right\}$. If $J \varsubsetneqq I$ are ideals of $C(X)$ with $Z(J)=Z(I)$ and if $I$ is semiprime, there is an ideal $K$ strictly between $J$ and $I$. If $I$ and $J$ are $Z$-ideals, $K$ can be chosen to be of the form $P \cap I, P$ a prime ideal of $C(X)$. The maximal ideals of a semiprime ideal $I$ of $C(X)$ are of the form $I^{q} \cap I, q \in \beta X-Z(I)$. If $A \subset \beta X$ is closed, $I^{A}$ is a finitely generated ideal iff $A$ is open.
\end{abstract}

Let $X$ be a completely regular Hausdorff space and let $F$ denote the real or complex numbers. An examination of the ideal structure of $C(X)$, the $F$-algebra of continuous mappings from $X$ to $F$, along the lines begun in [2] is continued here. For a subset $A$ of $\beta X$, the Stone-Čech compactification of $X$, let $I^{A}\left(F^{A}\right)$ denote the ideal of functions $f$ in $C(X)$ for which $A \subset \operatorname{cl}_{\beta X} Z(f)\left(A \subset\right.$ int $\left._{\beta X} \operatorname{cl}_{\beta X} Z(f)\right)$ where $Z(f)=\{x \in X: f(x)=0\}$. Every ideal of $C(X)$ lies between $I^{A}$ and $F^{A}$ for appropriate $A$ (1.3), so that the ideal structure of $C(X)$ can be examined piecewise: it is enough to obtain information about the ideals of the algebras $I^{A} / F^{A}, A \subset \beta X$. If $A \subset X$, $I^{A}\left(F^{A}\right)$ is just the ideal of maps vanishing on $A$ (on a neighborhood of $A$ ). This means the results of [2] apply to suggest that $I^{A} / F^{A}$ is likely to be algebraically complicated (and that the piecewise approach will not be dramatically simpler than the global one). This suspicion is confirmed here. In fact the qualitative complexity of $I^{A} / F^{A}$ is shown to be substantially independent of the topological structure of $A$ : For any $A \subset \beta X, I^{A} / F^{A}$ fails to contain either maximal or minimal ideals, and unless it vanishes, $I^{A} / F^{A}$ has infinite Krull dimension.

Because of this, we are led to examine more closely what can occur between two ideals of $C(X)$ which both lie between $F^{A}$ and $I^{A}$. For a subset $J$ of $C(X)$, set $Z(J)=\bigcap\left\{\mathrm{cl}_{\beta X} Z(f): f \in J\right\}$. If $J \varsubsetneqq I$ are ideals of $C(X)$ with $Z(J)=Z(I)$ and if $I$ is

Received by the editors January $9,1970$.

AMS subject classifications. Primary 4625; Secondary 1320, 0685.

Key words and phrases. Completely regular space, algebra of continuous functions, Stone-Čech compactification, prime ideal, $Z$-ideal, maximal ideal, minimal ideal, Krull dimension, maximal algebra, minimal algebra.

(') Author is a National Science Foundation Graduate Fellow.

Copyright (C) 1970, American Mathematical Society 
semiprime, we can find an ideal of $C(X)$ strictly between $I$ and $J$ (compare [12, 7.7.2, p. 183]). This means if $J$ is an ideal strictly between $F^{A}$ and $I^{A}$ there are ideals $J$ and $\bar{J}$ so that

$$
F^{A} \subset \underline{J} \subset J \subset \bar{J} \subset I^{A}
$$

with all inclusions proper (4.5). By repeating the process we conclude that through any such $J$ we can thread infinite ascending and descending chains of ideals lying between $F^{A}$ and $I^{A}$. As an application, we show that for a semiprime ideal $I$, $q \rightarrow I^{q} \cap I$ is a one-to-one correspondence between $\beta X-Z(I)$ and the set of maximal ideals of $I$. This has been obtained in [6] for $I=I^{p}$ or $F^{p}, p \in \beta X$.

Needless to say, the topology on $X$ is relevant to the structure of $C(X)$. For example, whether $F^{A}$ and $I^{A}$ coincide depends topologically on $A$. (If $X$ is discrete, this happens for every $A$, so that the ideal structure of $C(X)$ is completely known in this case.) In this direction, we show if $A \subset \beta X$ is closed, $I^{A}$ is finitely generated as an ideal of $C(X)$ iff $A$ is open (compare [2, Corollary 2, p. 176]). This means if $X$ is connected, $I^{A}$ is finitely generated only when it is trivial or all of $C(X)$.

The proof of many of these results is accomplished by exploiting techniques first developed by C. W. Kohls [7], [8] and first used to exhibit an abundance of prime ideals in $C(X)$. As existence theorems, our results are positive, but they conclusively place $C(X)$ outside the range of finitistic algebra. For the analyst, perhaps, they serve to reinforce the prevailing tendency to concentrate only on the structure of closed ideals in Banach algebras. To do otherwise, even for primeval $C[0,1]$, is to open Pandora's box.

1. Preliminary results. We shall view $X$ as a subspace of $\beta X$. For $A \subset \beta X$, we will abbreviate $\operatorname{cl}_{\beta X} A$, the closure of $A$ in $\beta X$, to $\operatorname{cl} A$ or $\bar{A}$ as convenient.

LEMMA 1.1. $f \in F^{A}$ iff there is a neighborhood $V$ of $A$ for which $V \cap X \subset Z(f)$.

Proof. Because $X$ is dense in $\beta X, V \cap X$ is dense in int $_{\beta X} V$ and the condition is sufficient. If $f \in F^{A}, Z(f)=X \cap \mathrm{cl} Z(f)$, so that we obtain the condition with $V=\operatorname{cl} Z(f)$.

If $A \subset X$, let $I_{A}\left(F_{A}\right)$ denote the ideal of maps vanishing on $A$ (on a neighborhood of $A$ ). An ideal $I$ is called a $Z$-ideal if every function whose zero set agrees with that of some function in $I$ is also in $I$.

Proposition 1.2. $I^{A}$ and $F^{A}$ are Z-ideals of $C(X)$ and if $A \subset X, I^{A}=I_{A}$ and $F^{A}=F_{A}$.

Proof. The statements involving $F^{A}$ follow directly from the above lemma. Suppose $f, g \in I^{A}$. Among the compactifications of $X$ it is characteristic of $\beta X$ that $\operatorname{cl} Z(f) \cap \operatorname{cl} Z(g)=\operatorname{cl}[Z(f) \cap Z(g)][5,6.5 \mathrm{IV}$, p. 86]. This means

$$
A \subset \operatorname{cl}[Z(f) \cap Z(g)] \subset \operatorname{cl} Z(f-g),
$$

and hence $f-g \in I^{A}$. If $f \in I^{A}, g \in C(X), A \subset \operatorname{cl} Z(f) \subset \operatorname{cl} Z(f g)$, so $I^{A}$ is an ideal. If $f \in C(X)$ and $Z(f)=Z(g)$ for some $g \in I^{A}, A \subset \operatorname{cl} Z(g)=\operatorname{cl} Z(f)$, so $I^{A}$ is a $Z$-ideal. Finally since $Z(f)=X \cap \operatorname{cl} Z(f), A \subset X$ implies $I^{A}=I_{A}$. 
The reason $I^{A}$ and $F^{A}$ are so valuable for the study of the ideal structure of $C(X)$ is the following.

TheOREM 1.3 (MCKNIGHT). If $J$ is an ideal of $C(X)$ and $A=Z(J), F^{A} \subset J \subset I^{A}$.

Proof. $Z(J)=\bigcap\{\operatorname{cl} Z(f): f \in J\}$, so that $J \subset I^{A}$ is clear. Let $g \in F^{A}$. By 1.1, there is an open neighborhood $U$ of $A$ with $U \cap X \subset Z(g)$. For each $y \in \beta X-U$ we can find an $f_{y} \in J$ so that $y \in \operatorname{cl} Z\left(f_{y}\right)$. Since $\beta X$ is regular we may choose a neighborhood $U_{y}$ of $y$ disjoint from $\mathrm{cl} Z\left(f_{y}\right)$. The $U_{y}$ 's cover the compact set $\beta X-U$ so for some $y_{1}, \ldots, y_{n} \in \beta X, \beta X-U \subset \bigcup_{i=1}^{n} U_{y_{i}}$. Set $f=\sum_{i=1}^{n} f_{y_{i}} \bar{f}_{y_{i}} \in J$. $Z(f)=\bigcap_{i=1}^{n} Z\left(f_{y_{i}}\right)$ so that $\operatorname{cl} Z(f)=\bigcap_{i=1}^{n} \operatorname{cl} Z\left(f_{y_{i}}\right)$ and hence

$$
\beta X-U \cap \operatorname{cl} Z(f) \subset \bigcup_{i=1}^{n} U_{y_{i}} \cap \bigcup_{j=1}^{n} \operatorname{cl} Z\left(f_{y_{i}}\right) \subset \bigcup_{i=1}^{n} U_{y_{i}} \cap \operatorname{cl} Z\left(f_{y_{i}}\right)=\varnothing .
$$

This means $Z(f)=X \cap \operatorname{cl} Z(f) \subset U \cap X \subset Z(g)$; that is, $Z(f) \subset$ int $_{X} Z(g)$. This allows us to define a continuous function $h$ by

$$
\begin{aligned}
h(x) & =g(x) / f(x), & & x \in X-\text { int }_{X} Z(g), \\
& =0, & & x \in Z(g) .
\end{aligned}
$$

But then $g=h f \in J$, so $F^{A} \subset J$ as required.

This means we can examine the ideals of $C(X)$ by fixing $A \subset \beta X$ and looking at the structure of $I^{A} / F^{A}$. Sometimes there is nothing to examine. For example, since $I^{A}=\bigcap_{p \in A} I^{p}$ and $F^{A}=\bigcap_{p \in A} F^{p}$, if $X$ is a $P$-space $[5,4 \mathrm{~L}$, p. $63 ; 7 \mathrm{~L}$, p. 111$]$, $I^{A}=F^{A}$ for every $A \subset \beta X$. Examples include discrete spaces and there are others $[5,4 \mathrm{~N}$, p. 64].

Corollary 1.4. If $X$ is discrete, every ideal of $C(X)$ is of the form $I^{A}, A \subset \beta X$.

With the compact-open topology, $C(X)$ becomes a locally $m$-convex topological algebra and its continuous dual can be identified with the regular Borel measures on $X$ of compact support [3, Theorem 1(iii), p. 203]. The next result shows, however, that these measures are oblivious to any algebraic subtleties which may occur between $F^{A}$ and $I^{A}$.

THEOREM 1.5. $F^{A}$ is compact-open dense in $I^{A}$.

Proof. Let $C^{*}(X)$ denote the subalgebra of $C(X)$ consisting of bounded continuous functions. For $B \subset C(X)$ set $B^{*}=B \cap C^{*}(X)$. Let $K \subset X$ be compact. First we show $I_{\mid K}^{A^{*}}=I_{\mid K}^{A}$. Let $f \in I^{A} . f=|f|^{1 / 2}\left(|f|^{1 / 2}\right.$ sign $\left.f\right)$ where $(\operatorname{sign} f)(x)=$ $f(x) /|f(x)|$ if $f(x) \neq 0$, so $f=g h$ for some $g, h \in I^{A}$ with $h \geqq 0 . g \mid K$ extends to some $\tilde{g} \in C^{*}(X)$ [3, Lemma 1(i), p. 201]. If $M$ is the maximum of $h$ on $K, h \wedge M \in I^{A^{*}}$ because $Z(h \wedge M)=Z(h)$ and $I^{A}$ is a $Z$-ideal. Hence $\tilde{g}(h \wedge M) \in I^{A^{*}}$, and this function agrees with $f$ on $K$. Since the compact-open topology is just the topology of uniform convergence on the compact parts of $X$, it is now sufficient to show $F^{A^{*}}$ is sup norm dense in $I^{A^{*}}$. Let $f \in I^{A^{*}}, \varepsilon>0$. Since $f$ is bounded, it has an 
extension $\tilde{f} \in C(\beta X) . A \subset \mathrm{cl} Z(f) \subset Z(\tilde{f})$, so that $U=\tilde{f}^{-1}(\mathrm{cl}(B(0, \varepsilon / 2)))$ is a compact neighborhood of $A$ disjoint from the closed set $L=\{y \in \beta X:|\tilde{f}(y)| \geqq \varepsilon\}$. Since $\beta X$ is normal, there is an $\tilde{h} \in C(\beta X)$ with $0 \leqq \tilde{h} \leqq 1, \tilde{h} \mid U=0$ and $\tilde{h} \mid L=1$. If we set $h=\tilde{h} \mid X, U \cap X \subset Z(h)$, so that $f h \in F^{A^{*}}$ and yet $\|f-f h\|_{X} \leqq\|\tilde{f}(1-\tilde{h})\|_{\beta X} \leqq \varepsilon$.

Lemma 1.6. $Z\left(F^{A}\right)=\bar{A}=Z\left(I^{A}\right)$ and if $F^{A} \subset I^{B}, \bar{B} \subset \bar{A}$.

Proof. The second assertion follows from the first since $F^{A} \subset I^{B}$ implies $Z\left(I^{B}\right)$ $\subset Z\left(F^{A}\right)$. Surely $\bar{A} \subset Z\left(I^{A}\right) \subset Z\left(F^{A}\right)$ so it suffices to show that $Z\left(F^{A}\right) \subset \bar{A}$. If $q \notin \bar{A}$, there is an $\tilde{f} \in C(\beta X)$ which vanishes on a neighborhood of $\bar{A}$ but not at $q$. $f=\tilde{f} \mid X \in F^{A}$ and yet $q \notin \operatorname{cl} Z(f)$, since $\operatorname{cl} Z(f) \subset Z(\tilde{f})$. So $q \notin Z\left(F^{A}\right)$.

The standard reference on $C(X)$ is [5]. However only real-valued functions are considered there, and we must on occasion introduce ad hoc arguments to handle the complex case as well. An example is the following.

THEOREM 1.7 (GELFAND-KOLMOGOROFF). $q \rightarrow I^{q}$ is a one-to-one correspondence between $\beta X$ and the set of maximal ideals of $C(X)$.

Proof. For $B \subset C(X)$ let $B_{r}$ denote the intersection of $B$ with $C_{r}(X)=C(X, R)$, the real-valued continuous functions on $X$ (of course if $F=R, B_{r}=B$ ). Let $Z_{F}: C(X, F) \rightarrow \mathscr{P}(X)$ be the $\operatorname{map} Z_{F}(f)=\{x \in X: f(x)=0\}$.

Suppose $M$ is a maximal ideal of $C(X)$. We claim $\mathscr{A}=Z_{F}(M)$ is a real $z$-ultrafilter on $X$ (see [5, 2.5, p. 26] for a definition). Since $Z(f)=Z\left(|f|^{2}\right)=Z(f \bar{f}), \mathscr{A}=$ $Z_{R}\left(M_{r}\right)$, and since $M_{r}$ is a proper ideal of $C_{r}(X), \mathscr{A}$ is a real $z$-filter on $X[5,2.3$, p. 25]. Suppose $\mathscr{F}$ is a real $z$-filter on $X$ which contains $\mathscr{A}$. $M \subset Z_{F}^{-1}(\mathscr{A}) \subset Z_{F}^{-1}(\mathscr{F})$, and exactly as in $[5,2.3]$, one shows that $Z_{F}^{-1}(\mathscr{F})$ is a proper ideal of $C(X)$. Thus $M=Z_{F}^{-1}(\mathscr{F})$ and so $\mathscr{A}=Z_{F}(M)=Z_{F}\left(Z_{F}^{-1}(\mathscr{F})\right)=\mathscr{F}$. Hence $\mathscr{A}$ is a real $z$ ultrafilter on $X$ and $M_{r}=Z_{R}^{-1}(\mathscr{A})$ is a maximal ideal of $C_{r}(X)[5,2.5$, p. 27].

By the usual Gelfand-Kolmogoroff Theorem [5, 7.3, p. 102] $M_{r}=I_{r}^{q}$ for some $q \in \beta X$. But if $f \in M, f \bar{f}=|f|^{2} \in M_{r}$, so $q \in \operatorname{cl} Z\left(|f|^{2}\right)=\operatorname{cl} Z(f)$ and $M \subset I^{q}$. By maximality, $M=I^{q}$. Conversely, $I^{q}$ is a proper ideal of $C(X)$ so it is contained in some maximal ideal $M$. By the above $M=I^{p}$ for some $p \in \beta X . F^{q} \subset I^{q} \subset I^{p}$ and 1.6 then forces $p=q$, so that $I^{q}=M$ is maximal. Finally, we conclude from 1.6 that the correspondence is one-to-one.

As a sample application we have

CoROllaRy $1.8[5,2.11$, p. 29]. Every proper prime ideal of $C(X)$ is contained in a unique maximal ideal.

Proof. Every proper prime $P$ is contained in some maximal ideal. If $P \subset I^{q}$, then $F^{q} \subset P$. For if $g \in F^{q}$, choose $\tilde{f} \in C(\beta X)$ so that $\tilde{f}(q) \neq 0$ and

$$
\operatorname{supp} \tilde{f} \subset \operatorname{int}_{\beta X} \mathrm{cl}_{\beta X} Z(g) \text {. }
$$

Then $f=\tilde{f} \mid X \notin I^{q}$ yet $f g=0 \in P$. Hence the Gelfand-Kolmogoroff Theorem and 1.6 guarantee that $P$ can be contained in at most one maximal ideal. 
LeMma 1.9. If $g \in C(X)-F^{A}, g \notin g I^{A}+F^{A}$.

Proof. Suppose instead that $g=g f+h$ for some $f \in I^{A}, h \in F^{A}$. $U \cap X \subset Z(h)$ for some neighborhood $U$ of $A$, so that on $U \cap X, g=g f$. In fact, $|g|=|g|(|f| \wedge 1)$ on $U \cap X$, since if $g(x) \neq 0,|f(x)|=|g(x) / g(x)|=1$. But $|f| \wedge 1$ has an extension $\tilde{f}^{\prime} \in C(\beta X)$ and since $|f| \wedge 1 \in I^{A^{*}}, V=U \cap \tilde{f}^{\prime-1}(B(0,1 / 2))$ is a neighborhood of $A$ in $\beta X$. Because $g \notin F^{A}, 1.1$ guarantees that $g(x) \neq 0$ for some $x \in V \cap X$. But then $f^{\prime}(x)=|f(x)| \wedge 1=|g(x) / g(x)|=1$, which is a contradiction.

This means in particular that if $I^{A} / F^{A} \neq 0$, neither $I^{A} / F^{A}$ nor $I^{A}$ has an identity. For choose $g \in I^{A}-F^{A}$. If either has an identity, there is an $f \in I^{A}$ with $f g-g \in F^{A}$, and hence $g \in g I^{A}+F^{A}$ in violation of the lemma. So a distinction should be made between the ring and algebra ideals of these algebras (see 4.6, 4.7). Ideal will mean subgroup closed under multiplication from the ring and algebra ideal will mean subspace closed under multiplication from the ring. Of course if $J$ is an ideal of $C(X)$ lying between $F^{A}$ and $I^{A}$, it is an algebra ideal of $I^{A}$ and $J / F^{A}$ is an algebra ideal of $I^{A} / F^{A}$.

Lemma 1.9 allows us to prove the next three assertions exactly as Theorem 1 , Corollary 1 and Theorem 2, respectively, are proved from the lemma in [2]. 1.9 also holds for bounded functions: if $g \in C^{*}(X)-F^{A^{*}}, g \notin g I^{A^{*}}+F^{A^{*}}$, and we could again formally use the proofs of [2] to obtain the following for $I^{A^{*}} / F^{A^{*}}$ instead of $I^{A} / F^{A}$.

THEOREM 1.10. $I^{A} / F^{A}$ has no minimal (algebra) ideals. In fact if $I$ is a nonzero ideal of $I^{A} / F^{A}$, there is an ideal $J$ of $C(X)$ lying between $F^{A}$ and $I^{A}$ such that $0 \varsubsetneqq J / F^{A} \varsubsetneqq I$.

COROLlary 1.11. $I^{A} / F^{A}$ has the descending chain condition on ideals of the form $J / F^{A}, J$ an ideal lying between $F^{A}$ and $I^{A}$ iff $I^{A} / F^{A}=0$.

Proposition 1.12. $I^{A} / F^{A}$ has the ascending chain condition on ideals of the form $J / F^{A}, J$ an ideal lying between $F^{A}$ and $I^{A}$ iff $I^{A} / F^{A}=0$.

One of the highlights of this paper is the stronger version of 1.12 found in 4.4 but much more than Lemma 1.9 is required to obtain it.

2. Finitely generated ideals of $C(X)$. Theorem 3 and its corollaries in [2] are partially generalized here. For $B \subset C(X)$ set $\operatorname{Re} B=\{\operatorname{Re} f: f \in B\}$ and $\operatorname{Im} B=$ $\{\operatorname{Im} f: f \in B\}$ (of course if $F=R, \operatorname{Im} B=0$ ). For an ideal $I$ of $C(X)$, set $B I=$ $\left\{\sum_{i} f_{i} g_{i}: f_{i} \in B, g_{i} \in I\right.$ with sums finite $\} . B I$ is an ideal of $C(X)$ contained in $I$. Finally, if $\operatorname{Re} B=B$ and $r$ is a positive rational with odd denominator in lowest terms, let $B^{r}=\left\{f^{r}(x)=f(x)^{r}: f \in B\right\}$.

THEOREM 2.1. Let $B \subset C(X)$ and let $J$ be an ideal of $C(X)$ for which $Z(J) \cap \bar{A}=\varnothing$. Suppose there is a $g \in I^{A}-F^{A}$ such that for each $f \in B$ we can find a neighborhood $U$ of $A$ and $a \delta>0$ so that $\delta|f| \leqq|g|$ on $U \cap X$. Then there is a prime ideal $P$ of $C(X)$ with

$$
\left[B I^{A}+F^{A}\right] \cap J \subset P \cap J \cap I^{A} \varsubsetneqq J \cap I^{A} .
$$


Proof. Since $B I^{A}+F^{A} \subset\left(B \cup\{g\}-F^{A}\right) I^{A}+F^{A}$, we may assume $\varnothing \neq B \subset C(X)$ $-F^{A}$. Define $\rho: R \rightarrow R$ by

$$
\begin{aligned}
\rho(y) & =1 / \log 2, & & y \geqq 1 / 2, \\
& =1 / \log 1 / y, & & 0<y<1 / 2, \\
& =0, & & y \leqq 0 .
\end{aligned}
$$

$\rho \in C(R), \rho(0)=0$, and by L'Hôpital's Rule, for each $a>0, \rho(y)^{a} / y \uparrow \infty$ as $y \downarrow 0$. Set $h=\rho \circ|g| \in I^{A}$. Since $Z(J)$ and $\bar{A}$ are disjoint closed sets in $\beta X$, they are contained in disjoint neighborhoods $S$ and $W$ and we may select a $t \in C(\beta X)$ which is 0 on $S$ and 1 on $W . t=\tilde{t} \mid X \in J$, by 1.3 , so that $s=t h \in J \cap I^{A}$. We claim $s^{a} \notin B I^{A}+F^{A}$ for each $a>0$. Suppose instead that

$$
s^{a}=\sum_{i=1}^{n} f_{i} g_{i}+k
$$

for some $a>0, f_{i} \in B, g_{i} \in I^{A}, 1 \leqq i \leqq n$, and $k \in F^{A}$. Choose an $\varepsilon(0<\varepsilon<1)$ so small that for $0<y<\varepsilon, \rho(y)^{a} / y \geqq 1$. Find a neighborhood $V$ of $A$ for which $V \cap X \subset Z(k)$. For each $1 \leqq i \leqq n$, there is a neighborhood $U_{i}$ of $A$ and a $\delta_{i}>0$ such that $\delta_{i}\left|f_{i}\right| \leqq|g|$ on $U_{i} \cap X$. Set $g_{i}^{\prime}=\left|g_{i}\right| \wedge \delta_{i} / n$ and $g^{\prime}=|g| \wedge 1$, and find extensions $\tilde{g}_{i}^{\prime}, \tilde{g}^{\prime} \in C(\beta X)$. Since the $\tilde{g}_{i}^{\prime \prime}$ 's and $\tilde{g}^{\prime}$ vanish on $A$,

$$
U=\bigcap_{i=1}^{n}\left[\tilde{g}_{i}^{\prime-1}\left(B\left(0, \delta_{i} / n\right)\right) \cap U_{i}\right] \cap V \cap W \cap \tilde{g}^{\prime-1}(B(0, \varepsilon))
$$

is a neighborhood of $A$. Because $g \notin F^{A}, 1.1$ gives us an $x \in U \cap X$ for which $g(x) \neq 0$. Because $\varepsilon<1, g^{\prime}$ and $|g|$ agree on $U \cap X$, so $0<|g(x)|<\varepsilon$. Yet $t(x)=1$ and for each $1 \leqq i \leqq n, \delta_{i}\left|f_{i}(x)\right| \leqq|g(x)|$ and $\left|g_{i}(x)\right|<\delta_{i} / n$, so we have

$1 \leqq \rho(|g(x)|)^{a} /|g(x)|=s^{a}(x) /|g(x)| \leqq \sum_{i=1}^{n}\left|g_{i}(x)\right|\left|f_{i}(x)\right| /|g(x)| \leqq \sum_{i=1}^{n}\left|g_{i}(x)\right| / \delta_{i}<1$.

This contradiction shows $s^{n} \in J \cap I^{A}-B I^{A}+F^{A}$ for each positive integer $n$. Since $\left[B I^{A}+F^{A}\right] \cap J$ is an ideal of $C(X)$, we may find a prime ideal $P$ of $C(X)$ containing it which is disjoint from the multiplicatively closed set $\left\{s^{n}: n=1,2,3, \ldots\right\}[5,0.16$, p. 6].

Observe that if $J=C(X), Z(J)=\varnothing$ so that 2.1 holds in this case for any $A \subset \beta X$. There are a number of situations in which the technical condition on $B$ is satisfied. For example, suppose $F^{A} \neq I^{A}$ and $B$ is locally a finite subset of $I^{A}$. That is, suppose for some finite subset $G \subset I^{A}$ every element of $B$ restricted to $X$ and some neighborhood of $A$ coincides in modulus with the restriction of some element of $G$ there. If $G=\left\{g_{1}, \ldots, g_{n}\right\}$ and $h \in I^{A}-F^{A}$, then set

$$
g=|h|+\sum_{i=1}^{n}\left|g_{i}\right| \in I^{A}-F^{A}
$$

The condition on $B$ in 2.1 is satisfied with this $g$. 
Or again suppose $F^{A} \neq I^{A}$ and suppose $B$ is countable. If $\bigcap Z_{F}(B) \in Z_{F}\left(I^{A}\right)$ then the conclusion of 2.1 also holds. For choose $h \in I^{A}-F^{A}$ and set

$$
g(x)=|h(x)|+\sum_{i=1}^{\infty} \frac{1}{2^{i}}\left(\left|f_{i}(x)\right| \wedge 1\right)
$$

where $\left\{f_{n}: n=1,2,3, \ldots\right\}$ is an enumeration of $B$ (with possible repetitions). Since by assumption

$$
Z(g)=Z(h) \cap \bigcap_{i=1}^{\infty} Z\left(f_{i}\right)=Z(h) \cap Z(k)=Z\left(|h|^{2}+|k|^{2}\right)
$$

for some $k \in I^{A}$, we have $g \in I^{A}$. Because $h \notin F^{A}, g \in I^{A}-F^{A} .\left|f_{i}\right| \wedge 1$ extends to some $f_{i}^{\prime} \in C(\beta X)$ and because $Z(k) \subset Z\left(f_{i}\right)=Z\left(\left|f_{i}\right| \wedge 1\right), f_{i}^{\prime}$ vanishes on $A$. So $U_{i}=f_{i}^{\prime-1}(B(0,1))$ is a neighborhood of $A$ and $\left(1 / 2^{i}\right)\left|f_{i}\right| \leqq|g|$ on $U_{i} \cap X$.

Under further restrictions on either $A$ or $B$ the condition $\cap Z_{F}(B) \in Z_{F}\left(I^{A}\right)$ holds automatically. For example if $B \subset I^{A}$ and $A \cap X$ is dense in $A$ (e.g. $A \subset X$ ), set $k=\sum_{i=1}^{\infty}\left(1 / 2^{i}\right)\left(\left|f_{i}\right| \wedge 1\right)$. $A \cap X \subset X \cap \operatorname{cl} Z\left(f_{i}\right)=Z\left(f_{i}\right)$ so $A \cap X \subset \bigcap_{i=1}^{\infty} Z\left(f_{i}\right)$ $=Z(k)$, and since $A \cap X$ is dense in $A, A \subset \operatorname{cl}_{\beta X} Z(k)$; that is, $k \in I^{A}$. For arbitrary $A \subset \beta X$, it is not necessarily true that $\bigcap Z_{F}(B) \in Z_{F}\left(I^{A}\right)$ (see 2.10). However if $Z_{F}(B)$ is a finite subset $\left\{Z\left(f_{i}\right), \ldots, Z\left(f_{n}\right)\right\}$ of $Z_{F}\left(I^{A}\right)$, then $k=\sum_{i=1}^{n} f_{i} \bar{f}_{i} \in I^{A}$ and $Z(k)=\bigcap_{i=1}^{n} Z\left(f_{i}\right)=\bigcap Z_{F}(B)$. From this last observation we select the following corollary.

Corollary 2.2. Suppose $F^{A} \neq I^{A}$ and $B$ is a finite subset of $I^{A}$. If $J$ is an ideal with $Z(J) \cap \bar{A}=\varnothing$, there is a prime ideal $P$ such that

$$
\left[B I^{A}+F^{A}\right] \cap J \subset P \cap J \cap I^{A} \varsubsetneqq J \cap I^{A} .
$$

Corollary 2.3. Let $X$ be a compact connected metric space. A proper ideal which is not contained in any nonmaximal prime cannot be countably generated.

Proof. Let $I$ be a proper ideal generated by a countable set $D . I$ is contained in a maximal ideal which, by 1.7 , must be of the form $I_{p}, p \in \beta X=X . D \subset I_{p}$ and $I=D C(X) \subset \operatorname{Re} D C(X)+\operatorname{Im} D C(X) \subset \operatorname{Re} D^{1 / 3} I_{p}+\operatorname{Im} D^{1 / 3} I_{p}\left(\right.$ since $\operatorname{Re} f=(\operatorname{Re} f)^{1 / 3}$ $\times(\operatorname{Re} f)^{2 / 3} \in \operatorname{Re} D^{1 / 3} I_{p}$, etc. $)=\left[\operatorname{Re} D^{1 / 3} \cup \operatorname{Im} D^{1 / 3}\right] I_{p}$. So if we let $B=\operatorname{Re} D^{1 / 3}$ $\cup \operatorname{Im} D^{1 / 3}, B$ is a countable set and $I+F_{p} \subset B I_{p}+F_{p}$. Because $X$ is a connected metric space, $F_{p} \neq I_{p}$. Applying the above remarks with $J=C(X)$, we obtain a prime ideal $P$ for which $I+F_{p} \subset P \cap I_{p} \subsetneq I_{p} . P$ is contained in some $I_{q}$, and because $F_{p} \subset P$, 1.6 forces $p=q$. Hence $I \subset I+F_{p} \subset P \varsubsetneqq I_{p}$; that is, $I$ is contained in a nonmaximal prime ideal.

TheOREM 2.4. Suppose $A=\operatorname{cl} Z(g)$ for some $g \in C(X)$. If $J$ is an ideal of $C(X)$ with $Z(J) \cap A=\varnothing$, and if $J \cap I^{A}$ is finitely generated, $A$ is open in $\beta X$.

Proof. Suppose $J \cap I^{A}$ is generated as an ideal of $C(X)$ by a finite set $D$. Then $D \subset I^{A}$ and $J \cap I^{A}=D C(X) \subset B I^{A}$ where $B$ is the finite set $\operatorname{Re} D^{1 / 3} \cup \operatorname{Im} D^{1 / 3}$. 
Hence $\left[B I^{A}+F^{A}\right] \cap J=J \cap I^{A}$ and by $2.2, F^{A}=I^{A}$. This means that $g \in F^{A}$ and int $A=$ int $_{\beta X} \operatorname{cl}_{\beta X} Z(g) \supset A \supset$ int $A$ so $A=$ int $A$ is open in $\beta X$.

Lemma 2.5. For each nonnegative $g \in C(X)$, there is a positive $u \in C(X)$ such that $u g=g \wedge 1$.

Proof. Set

$$
\begin{aligned}
u(x) & =1 / g(x), & & g(x) \geqq 1, \\
& =1, & & g(x) \leqq 1 .
\end{aligned}
$$

THEOREM 2.6. If $A \subset \beta X$ is closed, then $I^{A}\left[I^{A^{*}}\right]$ is finitely generated as an ideal of $C(X)\left[C^{*}(X)\right]$ iff $A$ is open.

Proof. Suppose $I^{A}$ is generated by a finite set $\left\{f_{1}, \ldots, f_{n}\right\}$. If $f \in I^{A}, f=\sum_{i=1}^{n} f_{i} g_{i}$ for some $g_{i} \in C(X), 1 \leqq i \leqq n$, so $\bigcap_{i=1}^{n} Z\left(f_{i}\right) \subset Z(f)$ and applying the characteristic property of $\beta X[5,6.5 \mathrm{IV}]$,

1.6 implies

$$
\bigcap_{i=1}^{n} \operatorname{cl} Z\left(f_{i}\right)=\operatorname{cl} \bigcap_{i=1}^{n} Z\left(f_{i}\right) \subset \operatorname{cl} Z(f) .
$$

$$
A=Z\left(I^{A}\right)=\bigcap_{i=1}^{n} \operatorname{cl} Z\left(f_{i}\right)=\operatorname{cl} \bigcap_{i=1}^{n} Z\left(f_{i}\right)=\operatorname{cl} Z(g)
$$

where $g=\sum_{i=1}^{n}\left|f_{i}\right|$. Applying 2.4 with $J=C(X)$ we see that $A$ is open in $\beta X$.

Suppose now that $I^{A^{*}}$ is generated in $C^{*}(X)$ by a finite set $D=\left\{g_{1}, \ldots, g_{n}\right\} \subset I^{A^{*}}$. Let $I$ be the ideal of $C(X)$ generated by $D$. Certainly $I \subset I^{A}$. If $f \in I^{A},|f|^{1 / 2} \in I^{A}$ and by 2.5 there is a positive $u \in C(X)$ such that $|f|^{1 / 2}=\sum_{i=1}^{n} f_{i}\left(u^{-1} g_{i}\right) \in I$. So $f=|f|^{1 / 2}\left(|f|^{1 / 2} \operatorname{sign} f\right) \in I$ and $I^{A}=I$ is finitely generated. By the above $A$ is open in $\beta X$, and the proof of one-half of the theorem is complete.

Conversely, suppose $A$ is open (closed) in $\beta X$. Then $A^{\prime}=A \cap X$ is open-closed in $X$, so $\chi_{A^{\prime}}$, the characteristic function for $A^{\prime}$, is continuous. Actually $\chi_{A^{\prime}} \in I^{A^{*}}$ since $A^{\prime}$ is dense in $A$. Thus $I^{A} \subset \chi_{A^{\prime}} I^{A} \subset \chi_{A^{\prime}} C(X) \subset I^{A}\left[I^{A^{*}} \subset \chi_{A^{\prime}} I^{A^{*}} \subset \chi_{A^{\prime}} C^{*}(X) \subset I^{A^{*}}\right]$, and $I^{A}\left[I^{A^{*}}\right]$ is generated as an ideal of $C(X)\left[C^{*}(X)\right]$ by $\chi_{A^{\prime}}$.

Since $\beta X$ is connected when $X$ is, we have as a consequence

Corollary 2.7. If $X$ is connected, an ideal of the form $I^{A}, A \subset \beta X$, is finitely generated only when it is trivial or all of $C(X)$.

Corollary 2.8. (Compare [2, Corollary 4, p. 177].) If $D \subset C(X)$ is finite, the ideal of $C(X)$ generated by $D$ is compact-open closed in $C(X)$ iff $\cap\{Z(f): f \in D\}$ is open in $X$.

Proof. Let $I$ be the ideal generated by $D$ and set $A=\bigcap\{Z(f): f \in D\}$ and $A^{\prime}=\bigcap\{\mathrm{cl} Z(f): f \in D\}$. Since $A^{\prime}=Z(I), F^{A^{\prime} \subset I \subset I^{A^{\prime}}}$.

If $I$ is compact-open closed in $C(X), I=I_{B}$ for some closed $B \subset X$ [3, Theorem 1(i)]. 1.6 implies $A^{\prime}=\bar{B}$, so $I=I_{B}=I^{\bar{B}}=I^{A^{\prime}}$ is finitely generated, and $A^{\prime}$ is open in $\beta X$ by 2.6. Since $A=\bigcap\{X \cap \operatorname{cl} Z(f): f \in D\}=X \cap A^{\prime}, A$ is open in $X$ as required. 
Suppose $A$ is open in $X$. Since $D$ is finite, $A=Z(f)$ and $A^{\prime}=\operatorname{cl} Z(f)$ for some $f \in I$. In particular $I \subset I^{A^{\prime}}=I_{A}$. If $g \in F_{A}, Z(g)$ is a neighborhood in $X$ of $Z(f)$ so $\left[5,1 D .1\right.$, p. 21] $g=f h \in I$ for some $h \in C(X)$. Thus $F_{A} \subset I \subset I_{A}$. Since $A$ is open in $X, F_{A}=I_{A}$ and $I=I_{A}$ is compact-open closed in $C(X)$.

Corollary 2.9. If $A$ is finite and $A-X$ is nonempty, $I^{A}$ is not finitely generated.

Proof. Otherwise, $A$ is open in $\beta X$ by 2.6 , and $A-X$ is also. Since $A-X$ is finite, each of its points is isolated in $\beta X$. But no point of $\beta X-X$ is isolated since $X$ is dense.

In view of [2, Corollary 2, p. 176] we might conjecture that 2.6 holds with "countably generated" in place of "finitely generated". This may be true. However the following shows that something other than 2.1 will be required to obtain it. A completely regular space is called hemicompact if it is the union of a sequence of compact parts $\left\{K_{n}\right\}$, and every compact set in $X$ is contained in some $K_{n}$. Examples include the locally compact, $\sigma$-compact spaces as well as the maximal ideal spaces of locally $m$-convex Fréchet algebras [11, 6.2(c), p. 25].

Proposition 2.10. Suppose $X$ is hemicompact and $A \subset \beta X$ is closed. Then it is true that $\cap Z_{F}(B) \in Z_{F}\left(I^{A}\right)$ for every countable $B \subset I^{A}$ iff $A \cap X$ is dense in $A$.

Proof. If $A \cap X$ is dense in $A, I^{A}=I_{A \cap X}$ is compact-open closed in $C(X)$. If $B=\left\{g_{n}\right\} \subset I^{A}$ is given, $g=\sum_{i=1}^{\infty}\left(1 / 2^{i}\right)\left(\left|g_{i}\right| \wedge 1\right)$ is the compact-open limit of a sequence on $I^{A}$. Thus $g \in I^{A}$ so that $\bigcap Z_{F}(B)=\bigcap_{i=1}^{\infty} Z\left(g_{i}\right)=Z(g) \in Z_{F}\left(I^{A}\right)$.

On the other hand, suppose $A \cap X$ is not dense in $A$. We first observe that $I^{A}$ is not compact-open closed in $C(X)$. For otherwise [3, Theorem 1i)], $I^{A}=I_{B}$ where $B=\bigcap\left\{Z(f): f \in I^{A}\right\}=X \cap Z\left(I^{A}\right)=X \cap A$; that is, $I^{A}=I_{X \cap A}$. Since $A \cap X$ is not dense in $A$, we may choose some $p \in A-\operatorname{cl} A \cap X$ and find an $\tilde{f} \in C(\beta X)$ which vanishes on a neighborhood of $\operatorname{cl} A \cap X$ but not at $p . f=\tilde{f} \mid X \in F_{A \cap X} \subset I_{A \cap X}=I^{A}$, whereas $p \notin \mathrm{cl} Z(f)$. This contradiction means $I^{A}$ is not compact-open closed in $C(X)$. Since $X$ is hemicompact, $C(X)$ is metrizable [1, Theorem 7, p. 486] and there is a $g \in C(X)-I^{A}$ and a sequence $\left\{f_{n}\right\}$ on $I^{A}$ with $f_{n} \rightarrow g$ uniformly of the compact parts of $X$. Set $h_{n}=\left|f_{n}\right| \wedge|g|$ and $B=\left\{h_{n}\right\}_{n=1}^{\infty}$. Since $Z\left(h_{n}\right)=Z\left(f_{n}\right) \cup Z(g), h_{n} \in I^{A}$. We observe that $h_{n}(x) \rightarrow|g(x)|$ pointwise. Because of this, $Z(g) \subset \bigcap_{i=1}^{\infty} Z\left(h_{i}\right) \subset Z(g)$ so in fact, $Z(g)=\bigcap Z_{F}(B)$. Since $I^{A}$ is a $Z$-ideal, this means $\bigcap Z_{F}(B)$ cannot be a member of $Z_{F}\left(I^{A}\right)$ without forcing $g \in I^{A}$, a contradiction.

In particular then, if $A \subset \beta X-X$, there is a countable set $B \subset I^{A}$ for which $\cap Z_{F}(B) \notin Z_{F}\left(I^{A}\right)$, and so 2.2 (and hence 2.6) does not follow from 2.1 for this $B$.

Notice also if $X$ is hemicompact but not compact, there is a sequence $\left\{\hat{J}_{n}\right\}$ on $C(X)$ for which $\mathrm{cl} \bigcap_{i=1}^{\infty} Z\left(f_{i}\right) \neq \bigcap_{i=1}^{\infty} \mathrm{cl} Z\left(f_{i}\right)$. For choose $q \in \beta X-X$ and use 2.10 to find a sequence $\left\{f_{n}\right\} \subset I^{q}$ for which $\bigcap_{i=1}^{\infty} Z\left(f_{i}\right) \notin Z_{F}\left(I^{A}\right)$. If it were true that $\operatorname{cl} \bigcap_{i=1}^{\infty} Z\left(f_{i}\right)=\bigcap_{i=1}^{\infty} \operatorname{cl} Z\left(f_{i}\right)$, then $q \in \operatorname{cl} Z(h)$ where $h=\sum_{i=1}^{\infty}\left(1 / 2^{i}\right)\left(\left|f_{i}\right| \wedge 1\right)$. This would force $\bigcap_{i=1}^{\infty} Z\left(f_{i}\right) \in Z_{F}\left(I^{q}\right)$, a contradiction. This means the characteristic zero-set property $[5,6.5 \mathrm{IV}]$ of $\beta X$ does not extend to countable intersections. 
3. Prime ideals in $I^{A} / F^{A}$. Let $S$ be a commutative algebra (perhaps without an identity). An (algebra) ideal $P$ of $S$ is prime if whenever $f g \in P, f, g \in S$, either $f \in P$ or $g \in P$. The Krull dimension of $S($ Krull $\operatorname{dim} S)$ is the length of the longest ascending chain of distinct proper prime algebra ideals of $S$. That is, Krull $\operatorname{dim} S$ $=\sup \left\{n-1:\right.$ there are $n$ prime algebra ideals $P_{i}$ in $S$ with $\left.P_{1} \varsubsetneqq P_{2} \varsubsetneqq \cdots \varsubsetneqq P_{n}\right\}$.

As is well known, $[9$, p. 220] algebras with identity, ascending chain condition and only one maximal ideal (so-called local Noetherian rings) have finite Krull dimension. Krull $\operatorname{dim} S$ is one of many numerical dimensions one can attach to $S$. All of these carry no simplifying structural information if they are infinite (or even large in some cases), but this itself is of interest. Thus the analyst, who tends to regard $C(X)$ philosophically as a primitive or irreducible (witness the GelfandNeumark Theorem), might welcome news that $C(X)$-even most pieces of $C(X)$ cannot be "represented" algebraically in any enlightening way. Such news is provided here. For we show that if $J \varsubsetneqq I$ are $Z$-ideals of $C(X)$ and $Z(J)=Z(I)$, the Krull dimension of $I / J$ is infinite. In particular if $F^{A} \neq I^{A}$,

$$
\text { Krull } \operatorname{dim} I^{A} / F^{A}=\infty .
$$

The proof utilizes machinery of Kohls [7], [8] which is summarized in [5, Chapters 5 and 14] and briefly reviewed here. The natural order on $R$ induces (pointwise) a partial order on $C_{r}(X)$ which is compatible with the algebraic structure. If $P$ is a proper prime ideal of $C_{r}(X), P$ is absolutely convex $(0 \leqq|f| \leqq|g|$ with $g \in P$ implies $f \in P)$ and the quotient map $C_{r}(X) \rightarrow C_{r}(X) / P$ transports this order to $C_{r}(X) / P$ and actually makes $C_{r}(X) / P$ a totally ordered ring $[5,5.5]$. Because of $1.8, C_{r}(X) / P$ has only one maximal ideal $I_{r}^{p} / P$ which must be the set of nonunits in $C_{r}(X) / P$.

This means that every element greater than a positive unit is a unit. For suppose instead that $0<a \leqq s, a, s \in C_{r}(X) / P$ with $a$ a unit and $s$ a nonunit. We may find $h, f \in C_{r}(X)$ with $f \in I_{r}^{p}$ such that $0 \leqq h \leqq f$ and $h+P=a, f+P=s$. Because $I_{r}^{p}$ is prime (and hence convex) $h \in I_{r}^{p}$, so $a=h+P \in I_{r}^{p} / P$ is a nonunit. Because $C_{r}(X) / P$ is totally ordered, this implies $a s<1$ for every $a, s \in C_{r}(X) / P, a$ a nonunit. In particular, for each positive nonunit $a, 0<a<1 / n, n=1,2, \ldots$

Since the prime ideals of $C_{r}(X) / P$ form a chain $\left.[5,14.3 \mathrm{c})\right]$, there is a smallest prime ideal $P^{a}$ in $C_{r}(X) / P$ which contains a given positive nonunit $a \in C_{r}(X) / P$. $a$ has a positive $n$th root $a^{1 / n}$, for if $a=f+P, f \geqq 0$, set $a^{1 / n}=f^{1 / n}+P$. For $b=g+P$, set $|b|=|g|+P$. Because $P^{a}$ consists of elements $b \in C_{r}(X) / P$ at least one of whose powers belongs to $a C_{r}(X) / P$, one can use the above remarks to show that

$$
P^{a}=\bigcup_{i=1}^{\infty}\left\{b \in C_{r}(X) / P:|b|<a^{1 / n}\right\} .
$$

An ideal $I$ is called semiprime if $f^{2} \in I$ implies $f \in I$. Examples include $Z$-ideals and, of course, prime ideals. The class of semiprime ideals is actually obtained by taking arbitrary intersections of primes $[10,4.16$, p. 69]. The following proposition 
provides the necessary device for utilizing Kohls' machinery in the complex as well as the real case.

LeMma 3.1. If $F=C$ and $I$ is a semiprime ideal of $C(X), I_{r}+i I_{r}=I$.

Proof. Recall $I_{r}=I \cap C_{r}(X)$. Clearly $I_{r}+i I_{r} \subset I$. Let $f \in I .|f|^{2}=f \bar{f} \in I_{r}$ and $I_{r}$ is a semiprime ideal of $C_{r}(X)$, so $|f|=\left(|f|^{1 / 2}\right)^{2} \in I_{r}$ and hence $|f|^{1 / 2} \in I_{r}$. $|f|^{1 / 2}(\operatorname{sign} f)$ is continuous so $f=|f|^{1 / 2}\left(|f|^{1 / 2} \operatorname{sign} f\right) \in I_{r} C(X) \subset\left[I_{r}+i I_{r}\right] C(X)=I_{r}+i I_{r}$, because $I_{r}+i I_{r}$ is an ideal.

THEOREM 3.2. Let I be a Z-ideal and $P$ a prime ideal of $C(X)$. If $I, P \subset I^{q}$ for some $q \in \beta X$ but $I \notin P$, then there is a prime ideal $Q$ of $C(X)$ containing $P$ for which

$$
P \cap I \varsubsetneqq Q \cap I \varsubsetneqq I .
$$

Proof. Choose $f \in I-P$. There is a positive $u \in C(X)$ for which $|f|^{2} u=|f|^{2} \wedge 1$ (2.5). If we set $h=u|f|^{2}, h$ lies in the $Z$-ideal $I$ and because $P$ is a proper prime and $u$ is a unit, $h \in I_{r}-P_{r}$. Because $0 \leqq h \leqq 1$,

$$
g(x)=\sum_{i=1}^{\infty} \frac{1}{2^{n}} h^{1 / n}(x)
$$

is continuous and since $Z(g)=Z(h), g \in I_{r}$. Let $a$ denote $h+P_{r}$ in the residue class ring $C_{r}(X) / P_{r}$. $P_{r}$ is a prime ideal of $C_{r}(X)$ and $a^{1 / n} \geqq 0$ in the order on $C_{r}(X) / P_{r}$. Actually since $h^{1 / n} \notin P_{r}, a^{1 / n}>0$. Because $h^{1 / n} \in I \subset I^{q}, a^{1 / n}$ is a positive nonunit in $C_{r}(X) / P_{r}$. So for all $n, m=1,2, \ldots, 0<a^{1 / n}<1 / m$.

Let $P^{a}$ be the smallest prime ideal of $C_{r}(X) / P_{r}$ which contains $a$. We claim $g+P_{r} \notin P^{a}$. For $g \geqq 2^{-2 n} h^{1 / 2 n}$ and $2^{-2 n}>a^{1 / 2 n}$, so that

$$
g+P_{r} \geqq 2^{-2 n}\left(h^{1 / 2 n}+P_{r}\right) \geqq a^{1 / 2 n} a^{1 / 2 n}=a^{1 / n} .
$$

Since this holds for all $n,\left(^{*}\right)$ implies that $g+P_{r} \notin P^{a}$.

There is a prime ideal $P^{\prime}$ of $C_{r}(X)$ containing $P_{r}$ such that $P^{\prime} \mid P_{r}=P^{a}$, and we have $g \in I_{r}-P^{\prime}$. If $F=R$, we set $Q=P^{\prime}$. Because $g \in I-Q \cap I$ and $h \in Q \cap I-P \cap I$, we have $P \cap I \varsubsetneqq Q \cap I \subsetneq I$ as required. If $F=C$, set $P^{\prime \prime}=P^{\prime}+i P^{\prime} . P^{\prime \prime}$ is an ideal of $C(X)$ and $h \in P^{\prime \prime} \cap I-P \cap I$. Because $P^{\prime}$ is prime, the multiplicatively closed set $S=\left\{g^{n}: n=1,2, \ldots\right\}$ is disjoint from $P^{\prime \prime}$, and we may find a prime ideal $Q$ containing $P^{\prime \prime}$ which is disjoint from $S[5,0.16]$. By 3.1, $P=P_{r}+i P_{r} \subset P^{\prime}+i P^{\prime}=P^{\prime \prime} \subset Q$. Because $g \in I-Q \cap I$ and $h \in Q \cap I-P \cap I$ we see that $P \cap I \varsubsetneqq Q \cap I \varsubsetneqq I$.

Lemma 3.3. Let I be an ideal of $C(X)$. If $K$ is a proper prime ideal of the ring $I$, there is a proper prime ideal $P$ of $C(X)$ such that $K=P \cap I$.

Proof. First observe that $K$ is an ideal of $C(X)$. For if $f \in C(X)$ and $p \in K$, $f p \in I$ and $(f p)^{2}=f(f p) p \in K$, so that $f p \in K$. Since $I-K$ is a nonempty multiplicatively closed set, there is a prime ideal $P$ of $C(X)$ disjoint from $I-K$ which contains $K$. Clearly $P \bar{P} \cap I=K$. 
THEOREM 3.4. If $J \varsubsetneqq I$ are ideals of $C(X)$ with $Z(J)=Z(I)$ and if $I$ is a Z-ideal, then the Krull dimension of $I / J$ is either 0 or $\infty$.

Proof. If Krull $\operatorname{dim} I / J \neq 0, I / J$ contains a proper prime ideal $Q . Q=K / J$ for some proper prime ideal of $K$ of $I$ containing $J$, and (3.3) $K=P \cap I$ for some proper prime ideal $P$ of $C(X) . P \subset I^{q}$ for some $q \in \beta X$. Set $A=Z(J)=Z(I)$. By $1.3, F^{A} \subset J$ $\subset K \subset I \subset I^{A} . F^{A} \subset K \subset P \subset I^{q}$ and 1.6 implies $q \in A$. Thus $I, P \subset I^{q} . I \notin P$ (otherwise $K=I$ ), and we apply 3.2 to obtain a prime ideal $P_{1}$ of $C(X)$ such that $K \varsubsetneqq P_{1} \cap I \varsubsetneqq I$. $Q_{1}=P_{1} \cap I / J$ is a proper prime algebra ideal of $I / J$ and $Q \varsubsetneqq Q_{1}$. We repeat this process on $Q_{1}$ to obtain a proper prime $Q_{2}$ with $Q \varsubsetneqq Q_{1} \varsubsetneqq Q_{2}$, and continuing this indefinitely, we obtain an ascending chain of distinct primes of $I / J$ of any desired length.

Corollary 3.5. If $J \varsubsetneqq I$ are Z-ideals of $C(X)$ and $Z(J)=Z(I)$, Krull $\operatorname{dim} I / J=\infty$.

Proof. Pick $h \in I-J$. Because $J$ is a $Z$-ideal, it is disjoint from

$$
S=\left\{h^{n}: n=1,2, \ldots\right\} .
$$

Choose a prime ideal $P$ of $C(X)$ containing $J$ and disjoint from $S . P \cap I / J$ is a proper prime ideal of $I / J$ so that Krull $\operatorname{dim} I / J>0$, and the result follows from 3.4. 1.6 now yields

COROllary 3.6. Unless it vanishes, $I^{A} / F^{A}$ has infinite Krull dimension.

4.9 below shows that the hypothesis $Z(J)=Z(I)$ in 3.4 is essential. And although 3.5 holds just as well if we assume only that $J$ is semiprime, we cannot replace “ $Z$-ideals" by "semiprime ideals" in 3.5. Consider for example, an upper (prime) ideal $I$ in $C_{r}(X)$ with immediate (prime) predecessor $J[5$, p. 200]. 0 is a proper prime in $I / J$ and if $0 \subset P \varsubsetneqq I / J$ is a prime, there is a prime $Q$ of $C_{r}(X)$ containing $J$ with $Q \cap I / J=P$. Since the prime ideals of $C_{r}(X)$ containing $J$ form a chain $\left.[5,14.3 \mathrm{c})\right]$, $Q \subset I$ or $I \subset Q . I \notin Q$, since $P \neq I / J$. Thus $J \subset Q \varsubsetneqq I$ and because $I$ is an upper ideal, $Q=J$ and $P=0$. So Krull $\operatorname{dim} I / J=1$.

Because of 3.6 it is sensible to examine topological conditions on $A$ which make $I^{A} / F^{A}$ nontrivial. There are a number of them. For example suppose $X$ is connected, $f$ is a nonzero nonunit in $C(X)$ and $A=Z(f)$ or $\operatorname{cl} Z(f)$. Then $f \in I^{A}-F^{A}$, since otherwise there is an open neighborhood $U$ of $A$ in $\beta X$ with $Z(f)=X \cap \operatorname{cl} Z(f)$ $\subset U \cap X \subset Z(f)$. This makes $Z(f)$ open-closed in $X$, and because $X$ is connected, this implies $f$ is either zero or a unit. If $X$ is metrizable, every closed set $A \subset X$ is of the form $Z(f), f \in C(X)$, so $I^{A} / F^{A}$ will then vanish only in the trivial cases $A=\varnothing$ or $X$. Although not every closed set in $\beta X$ is of the form $\operatorname{cl} Z(f), f \in C(X)$ (2.10), sets of this kind form a base for the closed sets in $\beta X$ [5, 6E.1, p. 94].

For a locally compact space $X$, let $C_{0}(X)\left[C_{c}(X)\right]$ denote the continuous functions $X \rightarrow F$ which vanish at $\infty$ [off a compact set]. If $X_{\infty}=X \cup\{\infty\}$ is the one-point compactification of $X, C_{0}(X)$ is $I^{\infty}$ and $C_{c}(X)$ is $F^{\infty}$ in $C\left(X_{\infty}\right)$. Yet if $X$ is not countably compact (or $\sigma$-compact but not compact), $C_{0}(X) \neq C_{c}(X)[5,7 \mathrm{G} .2$, p. 109], so we may apply 3.6 to obtain 
COROllary 3.7. If $X$ is locally compact but not countably compact (or $\sigma$-compact but not compact $), C_{0}(X)$ has infinite Krull dimension.

No point of $\beta X-X$ is of the form $\operatorname{cl} Z(f), f \in C(X)$. No $q \in \beta X-X$ can be of the form $Z(f), f \in C(\beta X)$, either; in fact, such sets contain a copy of the Stone-Čech compactification of the natural numbers $[5,9.5$, p. 132]. Nevertheless for some $X$, there are points $q \in \beta X-X$ with $I^{q} / F^{q} \neq 0$.

Proposition 3.8. If $X$ is a noncompact connected metric space, $I^{q} / F^{q}$ does not vanish for some $q \in \beta X-X$.

Proof. There is a proper countably infinite $D \subset X$ with no limit points in $X$. $D$ is closed in $X$, so $D=Z(f)$ for some $f \in C(X)$. Because $\beta X$ is compact, $q \in \operatorname{cl} Z(f)$ for some $q \in \beta X-X$. If $f \in F^{q}$, there is a neighborhood $U$ of $q$ with $U \cap X \subset$ $Z(f)=D$. Because $X$ is dense in $\beta X$ and $D$ has no limit point in $X, U \cap X$ is a nonempty open-closed set in $X$. So $X=U \cap X \subset D$, a contradiction. Thus $f \in I^{q}-F^{q}$.

Obviously this argument shows $I^{p} / F^{p} \neq 0$ for every $p \in \beta X$ which lies in the $\beta X$-closure of a fixed proper subset of $X$ which has no limit point in $X$. Not every point of $\beta X$ need be such a limit, but for $X=R$ and the sequence $N=\{1,2, \ldots\}$, $\operatorname{cl}_{\beta R} N=\beta N$ [5, p. 91] and card $\beta N=2^{c}$ [5, 90, p. 139]. Thus even for a fixed sequence, quite a few limit points can accumulate in this way.

Since $I^{A}=\bigcap_{p \in A} I^{p}$ and $F^{A}=\bigcap_{p \in A} F^{p}$, if $I^{p} / F^{p}$ vanishes for each $p \in A, I^{A} / F^{A}$ does also. The converse is false: if $X$ is a disconnected compact perfect metric space (e.g. a Cantor set), $I^{p} / F^{p} \neq 0$ for every $p$, yet $X$ contains a proper open-closed set $A$. However if $A \subset \beta X$ is finite, the converse holds. For if $f \in I^{p}-F^{p}, p \in A$, we may choose disjoint neighborhoods of $A-\{p\}$ and $p$ and find a $\tilde{g} \in C(\beta X)$ vanishing on the neighborhood of $A-\{p\}$ but not at $p$. Then $f(\tilde{g} \mid X) \in I^{A}-F^{A}$. So if $X$ is a noncompact connected metric space, there are finite sets $A \subset \beta X-X$ of every size for which $I^{A} / F^{A} \neq 0$.

Other conditions are possible. Call a point $y \in Y$ a $P$-point for $Y$ if $I_{y}=F_{y}$. If $C_{0}(X)=0$ (as happens if $X$ is a proper noncyclic subgroup of $R$ ), and if $q \in \beta X-X$ is a $P$-point for the space $\beta X-X$, then $I^{q}=F^{q}$. This follows from $[5,7 \mathrm{~F}$, p. 109]. This condition may not be very useful, since for the rationals $Q, C_{0}(Q)=0$ but $\beta Q-Q$ has no $P$-points [5, 60.5, p. 97]. If $T$ is a locally compact $\sigma$-compact space, the set of non- $P$-points for the space $\beta T-T$ is dense in $\beta T-T$ [5, 9M.3, p. 138], but it is not clear that $F^{q} \neq I^{q}$ when $q$ is a non-P-point for $\beta T-T$ (see [5, 6T.2, p. 99]).

4. Maximal ideals in $I^{A} / F^{A}$.

LEMma 4.1. Let I be a semiprime ideal of $C(X)$. Suppose $K \varsubsetneqq I$ and one of the following is true:

(a) $K$ is a maximal ideal of the ring $I$.

(b) $K$ is a maximal algebra ideal of the algebra $I$.

(c) $K$ is an ideal of $C(X)$ and there are no ideals of $C(X)$ strictly between $K$ and $I$.

Then $K=P \cap I$ for some proper prime ideal $P$ of $C(X)$. 
Proof. Because of 3.3 it is sufficient to show that in each case, $K$ is prime in the ring $I$. Observe first that $I^{2}$, the ideal of $C(X)$ generated by products $f g, f, g \in I$, is just $I$. For clearly $I^{2} \subset I$. Let $f \in I .|f|^{2}=f \bar{f} \in I$, so $|f| \in I$ and hence $|f|^{1 / 2} \in I$ and $|f|^{1 / 4} \in I . g=|f|^{1 / 2} \operatorname{sign} f \in C(X)$, so $h=|f|^{1 / 4} g \in I$ and hence $f=|f|^{1 / 4} h \in I^{2}$.

(a) If $K$ is not prime in $I$ there are $f, g \in I-K$ with $f g \in K$. Let $Z$ denote the integers. $Z f+I f+K$ and $Z g+I g+K$ are ideals of $I$ properly containing $K$, so by condition (a) they must both be $I$. Thus $I^{2}=(Z f+I f+K)(Z g+I g+K) \subset Z f g+I f g$ $+K f+K g+K^{2} \subset K$. Since $I=I^{2}$ we have a contradiction.

(b) Again if $K$ is not prime in $I$, there are $f, g \in I-K$ with $f g \in K$. Ff $+I f+K$ and $F g+I g+K$ are algebra ideals of $I$ properly containing $K$, so by condition (b) they are both $I$. Thus $I=I^{2} \subset F f g+I f g+K f+K g+K^{2} \subset K$, a contradiction.

(c) Finally, if $K$ is not prime in $I$ and $f, g \in I-K$ with $f g \in K, f C(X)+K$ and $g C(X)+K$ are ideals of $C(X)$ contained in $I$ but properly containing $K$, so by condition (c) they are both $I$. Again, $I=I^{2} \subset f g C(X)+K+K^{2} \subset K$, a contradiction.

THEOREM 4.2. Suppose $J \varsubsetneqq I$ are ideals of $C(X)$ with $Z(J)=Z(I)$. If I is semiprime, there is an ideal of $C(X)$ strictly between $J$ and $I$.

Proof. Suppose no such ideal exists. Then by 4.1(c), $J=P \cap I$ for some proper prime ideal $P$ of $C(X)$. In particular $J$ is semiprime, and this implies that there are no ideals $K$ of $C_{r}(X)$ strictly between $J_{r}$ and $I_{r}$. For otherwise, for $F=C$,

$$
J_{r}+i J_{r} \varsubsetneqq K+i K \varsubsetneqq I_{r}+i I_{r}
$$

and 3.1 implies $J \varsubsetneqq K+i K \varsubsetneqq I$.

We will now use the Kohls machinery to construct an ideal $K$ of $C_{r}(X)$ between $J_{r}$ and $I_{r}$ and obtain the required contradiction. Since $J_{r} \neq I_{r}$ we may choose some $f \in I_{r}-P_{r} . g=f^{2} \in I_{r}-P_{r}$ and we observe that $a=g+P_{r}$ is a positive nonunit in the totally ordered ring $C_{r}(X) / P_{r}$. For $P \subset I^{q}$ for some $q \in \beta X$ and since $J \subset P, q \in Z(J)$ $=Z(I)$ and thus $a \in I_{r}^{q} / P_{r}$, the set of nonunits of $C_{r}(X) / P_{r}$. Let $L$ denote the ideal generated by $a$. We claim $a^{1 / 2} \notin L$. If instead $a^{1 / 2}=s a$ for some $s \in C_{r}(X) / P_{r}, a^{1 / 2}$ is a nonunit so $s a^{1 / 2}<1$, and hence

$$
a^{1 / 2}=s a=\left(s a^{1 / 2}\right) a^{1 / 2}<a^{1 / 2},
$$

a contradiction. $a^{1 / 2}=g^{1 / 2}+P_{r}$, so if $N=p^{-1}(L)$ where $p: C_{r}(X) \rightarrow C_{r}(X) / P_{r}$ is the quotient map, $g^{1 / 2} \notin N$. Set $K=N \cap I_{r}$. Certainly

$$
J_{r}=P_{r} \cap I_{r} \subset N \cap I_{r}=K \subset I_{r} .
$$

Because $I_{r}$ is semiprime, $g^{1 / 2} \in I_{r}-K$. Since also $g \in K-J_{r}$, we have $J_{r} \varsubsetneqq K \varsubsetneqq I_{r}$, which is the desired contradiction.

4.2 holds in particular if $I$ is a $Z$-ideal, although we could derive it from 3.2 and 4.1 in this case. For a genuine example, consider $Q \varsubsetneqq P$ prime ideals of $C(X)$. The argument in 1.8 shows that $Z(Q)=Z(P)$, and 4.2 then yields an ideal between $P$ and $Q$--even in the (frequent) case when there are no prime ideals between them (see [5, p. 200]). 
Corollary 4.3. Suppose $J \varsubsetneqq I$ are ideals of $C(X)$ with $Z(J)=Z(I)$. If $I$ is semiprime, I/J has no maximal (algebra) ideals.

Proof. If $M$ is a maximal ideal in the ring $I / J, M=K / J$ for some maximal ideal $K$ in the ring $I$ which contains $J$. By 4.1(a), $K=P \cap I$ for some (prime) ideal $P$ of $C(X)$. In particular, $K$ is an ideal of $C(X)$. Since $J \subset K \varsubsetneqq I, Z(K)=Z(I)$ and applying 4.2 , we find an ideal $K^{\prime}$ of $C(X)$ strictly between $K$ and $I$. The ideal $K^{\prime} / J$ in the ring $I / J$ is proper and properly contains $M$, which is a contradiction.

Similarly, since $K^{\prime} / J$ is an algebra ideal of the algebra $I / J$, we may apply 4.1(b) and 4.2 to see that $I / J$ also has no maximal algebra ideals.

Corollary 4.4. (Compare [2, Theorem 4, p. 177].) $I^{A} / F^{A}$ has no maximal or minimal (algebra) ideals.

Proof. $Z\left(I^{A}\right)=\bar{A}=Z\left(F^{A}\right)$ and 4.3 implies the first part. The second is just 1.10 .

Corollary 4.5. If $J$ is an ideal of $C(X)$ strictly between $F^{A}$ and $I^{A}$, there are ideals $\underline{J}$ and $\bar{J}$ of $C(X)$ so that

$$
F^{A} \subset J \subset J \subset \bar{J} \subset I^{A}
$$

with all inclusions proper.

This follows from 4.2 and 1.10. The distinction implicit in 4.4 and 4.5 between algebra ideals, ring ideals and ideals of $C(X)$ lying between $F^{A}$ and $I^{A}$ is a real one.

Proposition 4.6. Every $f \in I^{A}-F^{A}$ is contained in an ideal of $I^{A}$ containing $F^{A}$ which is not an algebra ideal of $I^{A}$ (and hence not an ideal of $C(X)$ ).

Proof. $I=Z f+f I^{A}+F^{A}$ is an ideal of $I^{A}$ containing $f$ and $F^{A}$, and yet if $\alpha \in F$ is not an integer, $\alpha f \notin I$. For otherwise, $\alpha f=n f+f g+k, n \in Z, g \in I^{A}, k \in F^{A}$, so that

$$
(\alpha-n) f \in f I^{A}+F^{A} \text {. }
$$

Since $\alpha \neq n, f \in f I^{A}+F^{A}$ which contradicts 1.9 .

Proposition 4.7. Suppose $A$ is the zero-set of some $f \in C(X)$. Then every algebra ideal of $I_{A}$ lying between $F_{A}$ and $I_{A}$ is an ideal of $C(X)$ iff $\partial A$, the boundary of $A$ in $X$, consists of at most one point.

Proof. If $\partial A$ is empty, $A$ is open so that $F_{A}=I_{A}$. If $\partial A=\{p\}$ and $I$ is an algebra ideal of $I_{A}$ with $g \in I, k \in C(X)$, define $h \in I_{A}$ by

$$
\begin{aligned}
h(x) & =k(x)-k(p), & & x \in X-\operatorname{int}_{x} A, \\
& =0, & & x \in A .
\end{aligned}
$$

$h$ is well defined and continuous because $\partial A=\{p\} . g k-k(p) g=g[k-k(p)]=g h \in I$, and since $I$ is an algebra ideal, $g k \in I$ as required.

Conversely, suppose the algebraic condition is satisfied. $A=Z(f)$ for some $f \in C(X)$, and $J=F f+f I_{A}+F_{A}$ is an algebra ideal of $I_{A}$ containing $F_{A}$. By assumption, 
$g f \in J$ for each $g \in C(X)$. Thus for some $\alpha \in F, h \in I_{A}, k \in F_{A},(g-\alpha) f=f h+k$. Choose a neighborhood $U$ of $A$ in $X$ on which $k$ vanishes, and for each $p \in \partial A$, select a net $\left\{x_{\beta}\right\}$ on $U \cap X-A$ which converges to $p$. Since $f\left(x_{\beta}\right) \neq 0$ for all $\beta$,

$$
g\left(x_{\beta}\right)-\alpha=f\left(x_{\beta}\right) h\left(x_{\beta}\right) / f\left(x_{\beta}\right)=h\left(x_{\beta}\right) \rightarrow 0 .
$$

But $g\left(x_{\beta}\right) \rightarrow g(p)$, so $g(p)=\alpha$ for each $p \in \partial A$. That is, every continuous function on $X$ is constant on $\partial A$. This certainly implies $\partial A$ has at most one point.

Topological considerations can come into play in other ways. For example, if $A$ is a nonempty nondense open set in a connected metric space $X$, then $F^{A} / F^{\bar{A}} \neq 0$. (Here $\bar{A}$ denotes the closure of $A$ in either $X$ or $\beta X$ : since $\operatorname{cl}_{X} A \subset \mathrm{cl}_{\beta X} A$, we may assume $\bar{A}=\mathrm{cl}_{X} A$.) For choose $f \in C(X)$ with $Z(f)=\bar{A}$. Because $X$ is connected, $f \in I_{\bar{A}}-F_{\bar{A}}$ and since $I_{A}=F_{A}, f \in F_{A}-F_{\bar{A}}$. This has an interesting algebraic consequence because of the following.

Proposition 4.8. Unless it vanishes, $F^{A} / F^{\bar{A}}$ has no minimal or maximal (algebra) ideals, and its Krull dimension is infinite.

Proof. If $I$ is a nonzero ideal of $F^{A} / F^{\bar{A}}$ and $f+F^{\bar{A}} \in I-\{0\},\left\{I_{n}=f^{n} I^{A}+F^{\bar{A}} / F^{\bar{A}}\right\}_{n=1}^{\infty}$ is a descending sequence of distinct ideals of $F^{A} / F^{\bar{A}}$ containing $I$ (cf. 1.10). $Z\left(F^{\bar{A}}\right)$ $=Z\left(F^{A}\right)$ implies $F^{A} / F^{\bar{A}}$ cannot have a maximal ideal (4.3). Finally since $F^{A}$ and $F^{\bar{A}}$ are $Z$-ideals, 3.5 implies Krull $\operatorname{dim} F^{A} / F^{\bar{A}}=\infty$.

The next result has been obtained elsewhere for $I=I^{p}$ and $F^{p}, p \in \beta X$ [6].

THEOREM 4.9. If I is a semiprime ideal of $C(X)$, the map $q \rightarrow I^{q} \cap I$ is a one-to-one correspondence between $\beta X-Z(I)$ and the set of maximal (algebra) ideals of $I$.

Proof. It is well known [10, p. 49] that

$$
I / I \cap I^{q} \approx I+I^{q} / I^{q}, \quad q \in \beta X .
$$

If $q \notin Z(I), I^{q} \varsubsetneqq I+I^{q}$ and 1.7 implies that $I+I^{q}=C(X)$ and $I / I \cap I^{q}$ is a field. Thus $I^{q} \cap I$ is a maximal (algebra) ideal of $I$. If $p$ and $q$ are distinct points of $\beta X-Z(I)$, choose a neighborhood $U$ of $Z(I) \cup\{p\}$ not containing $q$ and find an $\tilde{f} \in C(\beta X)$ which vanishes on $U$ but not at $q . \tilde{f} \mid X \in I^{p} \cap I-I^{q} \cap I(1.3)$, so $q \rightarrow$ $I^{q} \cap I$ is $1-1$.

Finally, let $J$ be a maximal (algebra) ideal of $I$. 4.1 implies that $J=P \cap I$ for some proper prime ideal $P$ of $C(X) . F^{q} \subset P \subset I^{q}$ for some $q \in \beta X$ (cf. 1.8). If we set $A=Z(I)$,

$$
F^{A \cup(q)} \subset J=P \cap I \subset I^{A \cup\{q)},
$$

and hence $Z(J)=A \cup\{q\}$. This means $q \notin A$ since otherwise $Z(J)=Z(I)$, and we would contradict 4.2. But then $J=P \cap I \subset I^{q} \cap I \subsetneq I$ and by the maximality of $J$, $J=I^{q} \cap I$ as required.

In particular, if $A \subset \beta X$ is closed and $q \notin A$, there are no ideals between $I^{q} \cap I^{A}$ and $I^{A}$. Thus the condition $Z(J)=Z(I)$ in 3.4 and 4.2 is essential even when both $I$ and $J$ are $Z$-ideals. However, it is not known whether 4.2 holds if only this zero-set 
condition is assumed. For example, let $f$ be a nonnegative function vanishing only at a nonisolated point $p$ and set

$$
J=\bigcup_{a>1} f^{a} C(X) \text { and } I=f I_{p} .
$$

$J \varsubsetneqq I$ are ideals of $C(X)$ and although $I$ is not semiprime ( $f^{2} \in I$ but $f \notin I$ by 1.9), and although there are no ideals between $J$ and $I$ of the form $P \cap I, P$ prime, we can still construct an infinite ascending chain of ideals of $C(X)$ between $I$ and $J$. The easy but unenlightening proof is left for the interested reader.

\section{REFERENCES}

1. R. Arens, A topology for spaces of transformations, Ann. of Math. (2) 47 (1946), 480-495. MR 8, 165.

2. W. Dietrich, $A$ note on the ideal structure of $C(X)$, Proc. Amer. Math. Soc. 23 (1969), 174-178.

3. - The maximal ideal space of the topological algebra $C(X, E)$, Math. Ann. 183 (1969), 201-212.

4. L. Gillman, Countably generated ideals in rings of continuous functions, Proc. Amer. Math. Soc. 11 (1960), 660-666. MR 27 \#6120.

5. L. Gillman and M. Jerison, Rings of continuous functions, University Series in Higher Math., Van Nostrand, Princeton, N. J., 1960. MR 22 \#6994.

6. J. Keesling and P. Nanzetta, On certain ideals of $C(X)$, Notices Amer. Math. Soc. 16 (1969), 837. Abstract \#69T-B165.

7. C. W. Kohls, Prime ideals in rings of continuous functions, Illinois J. Math. 2 (1958), 505-536. MR 21 \#1518.

8. —-, Prime ideals in rings of continuous functions. II, Duke Math. J. 25 (1958), 447-458. MR 21 \#1519.

9. S. MacLane, Homology, Die Grundlehren der math. Wissenschaften, Band 114, Academic Press, New York and Springer-Verlag, Berlin, 1963. MR 28 \#122.

10. N. McCoy, Theory of rings, Macmillan, New York, 1964. MR 32 \#5680.

11. E. Michael, Locally multiplicatively-convex topological algebras, Mem. Amer. Math. Soc. No. 11 (1952). MR 14, 482.

12. W. Rudin, Fourier analysis on groups, Interscience Tracts in Pure and Appl. Math., no. 12, Interscience, New York, 1962. MR 27 \#2808.

NORTHWESTERN UNIVERSITY, EVANSTON, ILLINOIS 60201 